\title{
Dissolution of carbon dioxide bubbles and microfluidic multiphase flows
}

\author{
Ruopeng Sun and Thomas Cubaud* \\ Received 25th April 2011, Accepted 14th June 2011 \\ DOI: $10.1039 / \mathrm{c} 1 \mathrm{lc} 20348 \mathrm{~g}$
}

We experimentally study the dissolution of carbon dioxide bubbles into common liquids (water, ethanol, and methanol) using microfluidic devices. Elongated bubbles are individually produced using a hydrodynamic focusing section into a compact microchannel. The initial bubble size is determined based on the fluid volumetric flow rates of injection and the channel geometry. By contrast, the bubble dissolution rate is found to depend on the inlet gas pressure and the fluid pair composition. For short periods of time after the fluids initial contact, the bubble length decreases linearly with time. We show that the initial rate of bubble shrinkage is proportional to the ratio of the diffusion coefficient and the Henry's law constant associated with each fluid pair. Our study shows the possibility to rapidly impregnate liquids with $\mathrm{CO}_{2}$ over short distances using microfluidic technology.

\section{Introduction}

Carbon dioxide gas is widespread in natural and industrial processes. At the small scale, manipulating the dissolution of gaseous $\mathrm{CO}_{2}$ into liquids is important for managing a variety of carbonated liquid flows in micro-fuel cells ${ }^{1}$ and for better understanding reactive multiphase flows and carbon dioxide sequestration mechanisms in porous media. Microfluidic systems have yielded considerable insights into multiphase flows by facilitating the production and handling of individual bubbles and droplets. ${ }^{2-9} \mathrm{~A}$ common microfluidic flow pattern consists of elongated gas bubbles separated by liquid plugs. When the liquid wets the walls, ${ }^{10}$ bubbles assume a characteristic capsular shape, which fills most of rectangular cross-sections. The liquid generally fills the corners and gas slugs are separated from the walls by thin liquid films. ${ }^{11}$ Such segmented flows are advantageous for enhancing heat $\operatorname{transfer}^{12}$ and radial mixing ${ }^{13-15}$ due to the recirculation patterns in the liquid plugs between bubbles.

Typical microfluidic geometries used for the generation of monodisperse bubbles include T-junctions ${ }^{16-19}$ and focusing sections. ${ }^{20-25}$ In these systems, the bubble size is usually limited by the outlet channel width. Recently, the method of dissolving $\mathrm{CO}_{2}$ bubbles in a medium of various acidity ${ }^{26}$ was proposed as a route for the generation of ultra-small bubbles for biomedical applications. ${ }^{27}$ Although liquid-gas mass transfer has been previously investigated in bubble-columns ${ }^{28-30}$ and microchannels, ${ }^{31-34}$ the microfluidic flow behavior of bubbles having a time-dependent shape has remained mostly limited to thermally and chemically induced cavitation, ${ }^{35-38}$ particle-stabilized bubbles, ${ }^{39}$ and dissolving liquid droplets. ${ }^{40}$

Here, we investigate the interrelation between the dissolution of $\mathrm{CO}_{2}$ bubbles and microfluidic multiphase flows. We take

Department of Mechanical Engineering, Stony Brook University, Stony Brook, NY, 11794, USA. E-mail: thomas.cubaud@stonybrook.edu advantage of miniaturization to enhance liquid/gas mass transfer due to (a) the large surface-to-volume ratio of microbubbles and (b) the relatively large pressure required to displace fluids in microchannels. In addition, generating individual bubbles at the junction of a hydrodynamic focusing section allows for accessing very short time-scales after the fluids first contact. This technique is used to manipulate the rate of absorption of carbon dioxide gas into three low-viscosity solvents, namely water, ethanol, and methanol. We monitor the evolution of bubbles as they travel downstream and experience a significant reduction in size due to gas diffusion. In particular, we show that the rate of $\mathrm{CO}_{2}$ dissolution depends essentially on the liquid pair composition and the gas injection pressure. For short periods of time, "sharp" concentration gradients are maintained between the interface and the bulk liquid, leading to a fast gas impregnation process that is characterized by a bubble length decreasing linearly with time. For longer periods of time, the recirculation motion associated with the liquid plugs in segmented flows tends to homogenize the concentration profile of dissolved gas in the bulk liquid and bubble dissolution rate follows a normal diffusive behavior. We develop a simple model based on Fick's and Henry's laws to determine the initial rate of bubble shrinkage and the implication for microfluidic multiphase flows.

\section{Material and methods}

Hard microfluidic modules are fabricated in a clean room following standard microlithography and dry etching methods. ${ }^{23}$ Microchannels consist of etched-through silicon slides sandwiched between two glass pieces. These chips are useful for studying diffusive multiphase flows since they are not permeable to gases, do not deform under high-pressure, and permit a clear optical access for high-speed imaging (up to 20000 frames per second). The hydrodynamic section consists of a cross where gas is introduced in the central channel at a flow rate $Q_{\mathrm{G}}$ and liquid is 
injected in the side channels at a total flow rate $Q_{\mathrm{L}} \cdot{ }^{23}$ The flow features of liquid/gas mixtures are analyzed in the outlet channel having a height of $h=100 \mu \mathrm{m}$ and a width of $w=83 \mu \mathrm{m}$. The channel is nearly square in cross-section and has an aspect ratio $h / w \approx 1.2$. Liquid is injected in each side channel using a syringe pump and bone-dry $\mathrm{CO}_{2}$ gas is supplied from a compressed gas tank, the pressure of which is finely adjusted using a miniature regulator. The injection gas flow rate $Q_{\mathrm{G}}$ is measured with a gas volumetric flow meter and the injection pressure $P_{\mathrm{G}}$ is recorded using a differential pressure sensor embedded in the inlet gas line. As the gas flow path between the external pressure sensor and the microfluidic focusing junction is very small, we neglect the pressure drop along the injection gas line and use $P_{\mathrm{G}}$ as a control parameter. Experiments are performed at room temperature with pure water, ethanol, and methanol. Liquids are initially exposed to air resulting in a small initial $\mathrm{CO}_{2}$ concentration, which is negligible in comparison with the large concentrations reached during the experiment. A typical experiment consists of fixing the gas inlet pressure $P_{\mathrm{G}}$ and imposing the liquid flow rate $Q_{\mathrm{L}}$. Then, the corresponding injection gas flow rate $Q_{\mathrm{G}}$ is measured from the flow meter and a high-speed movie of the associated flow pattern is recorded.

\section{Bubbles formation}

The initial bubble size $d_{0}$ is defined immediately after bubble detachment from the continuous gas stream. Depending on the fluid pair composition and the injection pressure $P_{\mathrm{G}}$, a variety of flow patterns is observed in the outlet channel. Fig. 1 displays typical bubble formation processes for "weakly diffusive" $\left(\mathrm{CO}_{2}\right.$ and DI water) and for "strongly diffusive" $\left(\mathrm{CO}_{2}\right.$ and methanol) fluid pairs. In these micrographs, bubble production is steady, yet bubbles experience a continuous reduction in size $d(t, x)$, where $t$ is the time after breakup and $x$ is the distance from the junction, as they travel further downstream. The length of the liquid plugs between bubbles is denoted by $L$. Given the very large difference between liquid and gas densities, $\rho_{\mathrm{L}} / \rho_{\mathrm{G}} \approx O(3)$, the effective increase in liquid volume due to gas dissolution is negligible and $L$ remains constant along the outlet channel.

A critical parameter for describing multiphase dispersions, such as foams and emulsions, ${ }^{41}$ is the volume fraction of each component. For multiphase flows with negligible mass transfer and in the absence of significant compressibility effects, this quantity can be directly determined using the flow rates of

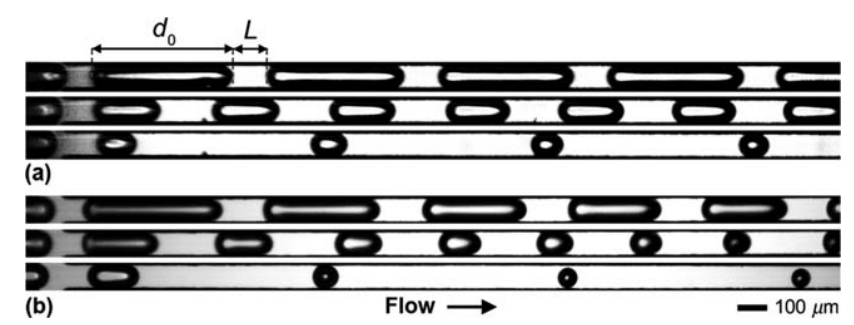

Fig. 1 Examples of diffusive multiphase flows. (a) Weakly diffusive bubbles $\left(\mathrm{CO}_{2}\right.$ in DI water), inlet gas pressure $P_{\mathrm{G}} \approx 20.4$ Psia, $\alpha_{\mathrm{L}}=0.23$, 0.47 , and 0.80 (from top to bottom). (b) Strongly diffusive bubbles $\left(\mathrm{CO}_{2}\right.$ in methanol), $P_{\mathrm{G}} \approx 17$ Psia, $\alpha_{\mathrm{L}}=0.24,0.51$, and 0.68 (from top to bottom). injection $Q_{\mathrm{L}}$ and $Q_{\mathrm{G}}$. For the case of a hydrodynamic focusing section with square microchannels of height $h$, a previous study ${ }^{23}$ has shown that the bubble size $d_{0}$ is related to the homogeneous liquid volume fraction $\alpha_{\mathrm{L}}=Q_{\mathrm{L}} /\left(Q_{\mathrm{L}}+Q_{\mathrm{G}}\right)$ according to $d_{0} / h \approx$ $1 / \alpha_{\mathrm{L}}$. This relationship can be deduced using a simple scaling argument that neglects the bubble end-caps curvature and liquid flow in the corners, the viscous contributions from the gas phase, and non-linear effects ${ }^{42}$ near breakup. The pitching time-scale $\tau$ is expected to scale with the liquid flow rate $Q_{\mathrm{L}}$ according to $\tau \approx$ $h / J_{\mathrm{L}}$, where the liquid superficial velocity $J_{\mathrm{L}}=Q_{\mathrm{L}} / h^{2}$. Assuming a constant bubble velocity during detachment and neglecting drift, the bubble velocity scales as $J_{\mathrm{B}} \approx\left(Q_{\mathrm{L}}+Q_{\mathrm{G}}\right) / h^{2}$. The pinching time-scale is also related to the bubble speed, such as $\tau \approx d_{0} / J_{\mathrm{B}}$, which overall yields the relationship $d_{0} / h \approx 1 / \alpha_{\mathrm{L}}$. Here, we measure the initial bubble size $d_{0}$ in our nearly square microchannels for the three test liquids and find good agreement with the expression

$$
d_{0} / w=c / \alpha_{\mathrm{L}}
$$

where the constant $c=1.5$ [Fig. 2(a)]. Although the constant $c$ is slightly larger than the channel aspect ratio $w / h \approx 1.2$, this scaling is very useful for estimating initial bubble sizes $d_{0} / w$ ranging between 1 and 30 . We also note that eqn (1) is independent from the liquid thermophysical properties, such as viscosity $\eta$ and surface tension $\gamma$. This behavior is assumed to remain valid as long as bubbles have nearly spherical front and rear end-caps with a curvature $\kappa \approx 2 / h$ and are not significantly deformed by viscous stresses, ${ }^{22}$ i.e., for small capillary numbers $\mathrm{Ca}=\eta J_{\mathrm{B}} / \gamma<10^{-2}$. Another parameter of interest is the liquid plug length $L$, which is deduced from eqn (1) using a volume conservation argument according to $L / w=c /\left(1-\alpha_{\mathrm{L}}\right)$. In Fig. 2
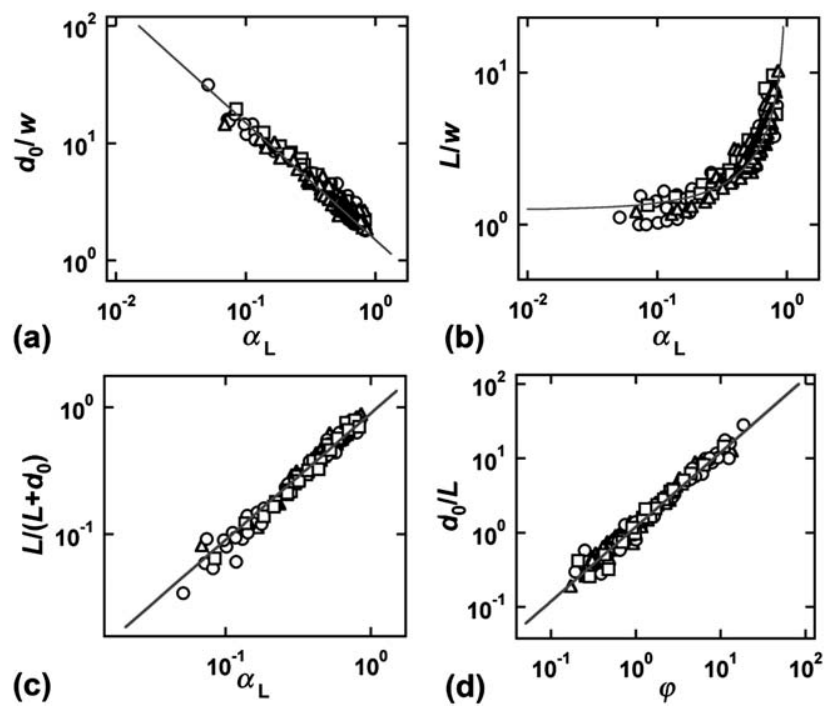

Fig. 2 Initial multiphase flow arrangement. $\mathrm{CO}_{2}$ bubbles in water $(\triangle)$, ethanol ( $\bigcirc)$, and methanol ( $\square$ ). (a) Initial bubble size $d_{0}$ as a function of liquid volume fraction $\alpha_{\mathrm{L}}$, solid line: $d_{0} / w=1.5 \alpha_{\mathrm{L}}{ }^{-1}$. (b) Liquid plug length $L$ versus $\alpha_{\mathrm{L}}$, solid line: $L / w=1.25 /\left(1-\alpha_{\mathrm{L}}\right)$. (c) Experimental liquid volume fraction $L /\left(L+d_{0}\right)$ versus injection liquid volume fraction, solid line $L /\left(L+d_{0}\right)=0.9 \alpha_{\mathrm{L}}$. (d) Initial bubble size normalized by liquid plug length $d_{0} / L$ versus flow rate ratio $\varphi=Q_{\mathrm{G}} / Q_{\mathrm{L}}$, solid line $d_{0} / L=1.2 \varphi$. 
(b), we measure $L$ and find that $c=1.25$ yields a good estimate, in particular for low liquid fraction $\alpha_{\mathrm{L}}<2 \times 10^{-1}$ when the volume of the bubble end-caps is comparable to the volume of a small liquid plug. The bubble size $d$ and liquid plug length $L$ allow for fully describing liquid/gas multiphase flows in compact channels. During bubble dissolution, the effective liquid fraction $\alpha_{\mathrm{L}}(x, t)$ is expected to rise due to the diffusion of $\mathrm{CO}_{2}$ into the liquid. To assess whether the experimental liquid fraction $\alpha_{\mathrm{L}, \mathrm{E}}(x, t)=$ $L /[L+d(x, t)]$, which can be computed from image processing, gives an accurate description of the evolving flow, we compare the initial experimental liquid fraction $\alpha_{\mathrm{L}, \mathrm{E}}=L /\left(L+d_{0}\right)$ with $\alpha_{\mathrm{L}}=Q_{\mathrm{L}} /\left(Q_{\mathrm{L}}+Q_{\mathrm{G}}\right)$ and find good agreement for $\alpha_{\mathrm{L}, \mathrm{E}}=0.9 \alpha_{\mathrm{L}}$ [Fig. 2(c)]. Many microbubble generation studies use the flow rate ratio $\varphi=Q_{\mathrm{G}} / Q_{\mathrm{L}}$ as a control parameter in the scaling of $d_{0}$ or $L$. Here, we show that the intrinsic ratio of bubble size to liquid plug length $d_{0} / L$ is directly proportional to the flow rate ratio $\varphi$ according to $d_{0} / L=1.2 \varphi$ [Fig. 2(d)]. In general, we find that the liquid or gas volume concentration $\left(\alpha_{\mathrm{L}}\right.$ or $\alpha_{\mathrm{G}}$, with $\alpha_{\mathrm{L}}+$ $\alpha_{\mathrm{G}}=1$ ) is useful to describe microbubble formation in confined geometries because this quantity is bound between 0 and 1 and gives an account of the resulting multiphase material. In summary, we find that the initial multiphase flow morphologies follow those of the non-diffusive case. This well-characterized system is used to examine the influence of pressure and fluid pair composition on the evolution of dissolving $\mathrm{CO}_{2}$ bubbles.

\section{Dissolution rate}

The gas dissolution has a strong effect on multiphase flow characteristics (Fig. 3). To measure the instantaneous bubble size $d(x, t)$ as a function of both the distance $x$ from the junction and the time $t$ after breakup, a time-space diagram is constructed from high-speed movies by plotting a line along the axis of the channel and applying the "reslice" function in ImageJ. The positions of the bubble front $x_{\mathrm{F}}(t)$ and rear $x_{\mathrm{R}}(t)$ are then numerically extracted from the diagram. This technique, which is similar to the one developed for analyzing droplet deformations

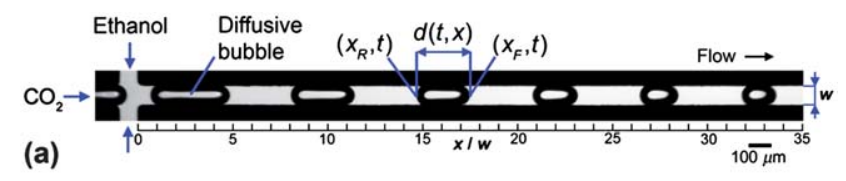

(a)

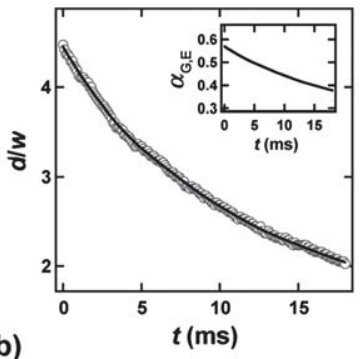

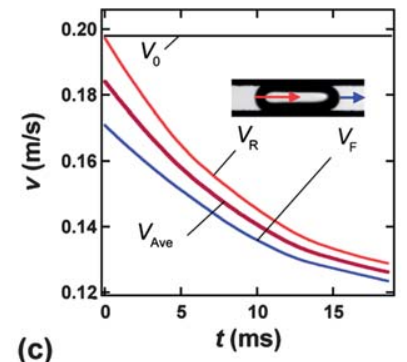

(c) in microchannels, ${ }^{43}$ allows us to automatically acquire bubble's front and rear edges and generate their spatial coordinates frame by frame, which is advantageous compared to manual scattered measurements. The bubble size evolution is calculated from the front and rear coordinates according to $d(t)=x_{\mathrm{F}}(t)-x_{\mathrm{R}}(t)$ [Fig. 3(b)]. The bubble average position is computed according to $x=\left[x_{\mathrm{F}}(t)+x_{\mathrm{R}}(t)\right] / 2$. We find, however, that the bubble temporal evolution $d(t)$ is more relevant for examining the influence of fluid properties and initial gas pressure $P_{\mathrm{G}}$ on dissolution processes. Indeed, the spatial position $x$ of a bubble at a given time $t$ depends on the bubble velocity, which is related to the initial flow rates. Using only the temporal evolution (i.e., the bubble reference frame) allows us to reduce the set of control parameters to the initial pressure $P_{\mathrm{G}}$. Bubble position data are typically smoothed using a Loess filter to obtain a mean curve [Fig. 3(b)]. The evolution of $d(t)$ also permits the computation of the gas fraction $\alpha_{\mathrm{G}, \mathrm{E}}(t)=d(t) /[d(t)+L]$, which decreases over time due to dissolution [Fig. 3(b), inset].

The velocity is calculated by differentiating the bubble spatial coordinates with time to obtain the bubble front velocity $V_{\mathrm{F}}=$ $d x_{\mathrm{F}} / d t$ and the bubble rear velocity $V_{\mathrm{R}}=d x_{\mathrm{R}} / d t$ [Fig. 3(c)]. We observe that a collapsing bubble elongated in a straight duct experiences a considerable difference between front and rear velocities $\left(V_{\mathrm{R}}>V_{\mathrm{F}}\right)$. The average bubble velocity $V_{\mathrm{Ave}}=\left(V_{\mathrm{F}}+\right.$ $\left.V_{\mathrm{R}}\right) / 2$ is typically smaller than the initial average multiphase velocity calculated based on the injection flow rates $V_{0}=\left(Q_{\mathrm{L}}+\right.$ $\left.Q_{\mathrm{G}}\right) /(h w)$. A consequence of the strong diffusion of $\mathrm{CO}_{2}$ in the liquid is a reduction in the mixture speed to compensate for the gas volume loss by diffusion, $Q_{\text {Diff }}(t)$. This effect is apparent when bubbles remain relatively large $(d / w>1)$ and are displaced at the average superficial multiphase velocity $J_{\mathrm{B}}(t) \approx$ $\left[Q_{\mathrm{L}}+Q_{\mathrm{G}}-Q_{\text {Diff }}(t)\right] /(h w)$. In long microchannels, diffusive segmented flows eventually transform into dilute bubbly flows that are characterized by small bubbles $(d / w<1)$ convected in the parabolic flow profile of the continuous phase. In this case, the bubble velocity is expected to be larger than the average mixture velocity.

We now focus on the rate of dissolution based on the bubble reference frame. Since our experiment is conducted at the millisecond range, we find it more practical to use the dimensional time $t$ rather than the dimensionless mixing time $t^{*}=t / t_{\mathrm{D}}$, with $t_{\mathrm{D}}=h w / D$, where $D$ is the diffusion coefficient. Indeed, the values of $t_{\mathrm{D}}$ are found in the second range with $t_{\mathrm{D}}=4.21 \mathrm{~s}$ for water, $t_{\mathrm{D}}=2.40 \mathrm{~s}$ for ethanol, and $t_{\mathrm{D}}=1.03 \mathrm{~s}$ for methanol, which does not yield considerable insight. The constants used in this work are displayed in Table 1.

The time evolution of normalized bubble lengths $d / w$ for a fixed gas inlet pressure $P_{\mathrm{G}}$ is shown in Fig. 4(a). For each set of control parameters, a bubble is chosen at random and its evolution is monitored using high-speed imaging. Data show that the initial bubble size $d_{0}$ and distribution do not significantly influence the initial diffusion rate since curves appear parallel. As the area of the thin films between the bubble and the walls is proportional to the bubble length $d$, this behavior suggests that the volume of gas lost by absorption in the thin films is negligible and mass transfer occurs predominantly through the bubble endcaps. To investigate the influence of the gas injection pressure $P_{\mathrm{G}}$, we plot the bubble evolution $d(t)$ for similar initial bubble size $d_{0}$ and three different inlet pressures [Fig. 4(b)]. It is evident from this figure that increasing $P_{\mathrm{G}}$ allows bubbles to dissolve

Fig. 3 Example of analysis of dissolving bubbles in microchannels. (a) Experimental micrograph for $\mathrm{CO}_{2} /$ ethanol fluid pair, $d_{0} / w=4.5, P_{\mathrm{G}} \approx$ 16 Psia. (b) Temporal evolution of bubble size $d / w$, symbol: raw data, solid curve: smoothed data. Inset: evolution of gas fraction calculated from $\alpha_{\mathrm{G}, \mathrm{E}}=d(t) /[d(t)+L]$. (c) Evolution of instantaneous velocities: bubble front velocity $\left(V_{\mathrm{F}}\right)$, bubble rear velocity $\left(V_{\mathrm{R}}\right)$, average velocity $\left[V_{\text {Ave }}=\left(V_{\mathrm{F}}+V_{\mathrm{R}}\right) / 2\right]$, and initial multiphase velocity $V_{0}$. 
Table 1 Reference diffusion coefficients $D$ and Henry's law constants $k_{\mathrm{H}}$ for carbon dioxide gas and the corresponding liquid

\begin{tabular}{llll}
\hline Constants & Water & Ethanol & Methanol \\
\hline$D / \mathrm{m}^{2} \mathrm{~s}^{-1}$ & $\begin{array}{l}1.97 \times 10^{-9} \\
(\text { ref. 44) }\end{array}$ & $\begin{array}{l}3.46 \times 10^{-9} \\
\text { (ref. 45) }\end{array}$ & $\begin{array}{l}8.02 \times 10^{-9} \\
\text { (ref. 46) }\end{array}$ \\
$k_{\mathrm{H}} / \mathrm{kPa}$ & $\begin{array}{l}167085 \\
(\text { ref. 45) }\end{array}$ & $\begin{array}{l}16009 \\
\text { (ref. 47) }\end{array}$ & $\begin{array}{l}14500 \\
\text { (ref. 48) }\end{array}$ \\
\hline
\end{tabular}
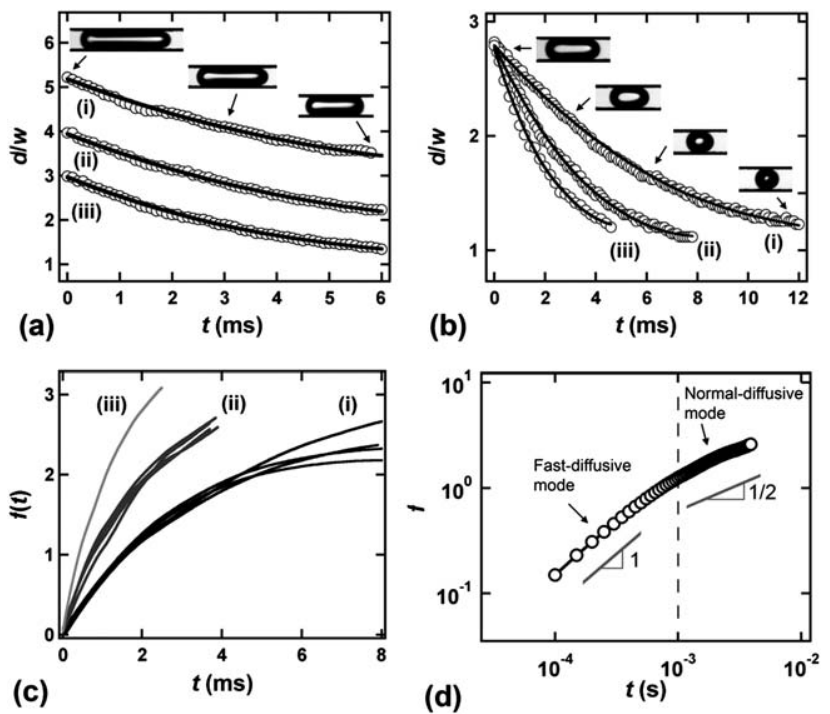

Fig. 4 Temporal evolution of bubble size. (a) Fixed inlet pressure, $P_{\mathrm{G}} \approx$ 17 Psia, (ethanol) $d_{0} / w=5.2$ (i), 3.9 (ii), and 2.9 (iii) (fluids: $\mathrm{CO}_{2} /$ ethanol). (b) Fixed initial bubble size $d_{0} / w=2.8, P_{\mathrm{G}} \approx 16$ (i), 17 (ii), and 18 Psia (iii) (fluids: $\mathrm{CO}_{2} /$ thanol). (c) Evolution of $f$ for various initial bubble sizes, $P_{\mathrm{G}} \approx 16$ (i), 17 (ii), and 18 Psia (iii) (fluids: $\mathrm{CO}_{2} /$ methanol). (d) Temporal evolution of $f$ showing a fast-diffusive mode $f \approx t$ for $t<10^{-3} \mathrm{~s}$ and a normal-diffusive mode $f \approx t^{1 / 2}$ for $t>10^{-3} \mathrm{~s}$ (fluids: $\mathrm{CO}_{2} /$ methanol).

faster. To perform a systematic analysis of experimental data, we introduce the function $f(t)$ such as

$$
f(t)=d_{0} / w-d(t) / w .
$$

The function $f(t)$ allows for removal of the dependence on the initial bubble size $d_{0}$. For instance, the functions $f$ associated with data displayed in Fig. 4(a) collapse on a single curve. For each fluid pair, the function $f$ is found to only depend on the initial pressure $P_{\mathrm{G}}[\mathrm{Fig} .4$ (c)]. In general, for short periods of time $(t<$ $\left.10^{-3} \mathrm{~s}\right), f$ is proportional to $t$, while for relatively large periods of time $\left(t>10^{-3} \mathrm{~s}\right), f$ is proportional to $t^{1 / 2}[\mathrm{Fig} .4(\mathrm{~d})]$. The exponent $1 / 2$ indicates a normal diffusion process characterized by a relatively smooth $\mathrm{CO}_{2}$ concentration gradient in the continuous phase. By contrast, for short periods of time, the concentration gradient is essentially localized in the thin interfacial region ${ }^{49}$ of width $\varepsilon \approx 1 \mathrm{~nm}$ between the liquid and gas phases and the system adopts a fast diffusive behavior.

For a variety of flow conditions, we fit $f$ with $f=a_{1} t$ for $t<10^{-3} \mathrm{~s}$, and $f=a_{2} t^{1 / 2}$ for $t>10^{-3} \mathrm{~s}$. The coefficients $a_{\mathrm{i}}$ depend on both the initial gas pressure $P_{\mathrm{G}}$ and the fluid pair. The coefficient $a_{1}$ is found to be a linear function of the initial gas pressure $P_{\mathrm{G}}[\mathrm{Fig}$. 5(a)]. The slope
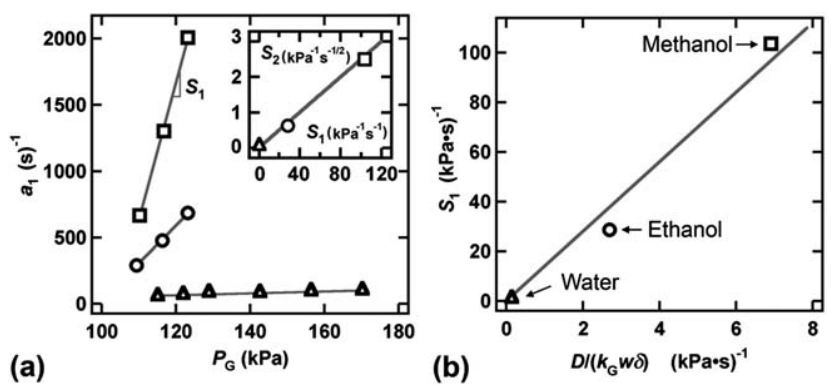

Fig. 5 Initial rate of dissolution for different fluid pairs: $\mathrm{CO}_{2} /$ water $(\triangle)$, $\mathrm{CO}_{2}$ /ethanol ( $\bigcirc$ ), and $\mathrm{CO}_{2} /$ methanol ( $\square$ ). (a) Evolution of the prefactor $a_{1}$ as a function of inlet gas pressure $P_{\mathrm{G}}$. Inset: evolution of $S_{2}$ as a function of $S_{1}$, solid line: $S_{2}=2.5 \times 10^{-2} S_{1}$. (b) Measured effective dissolution rate $S_{1}$ as a function of fluid properties. Solid line $S_{1}=$ $14 D /\left(k_{\mathrm{G}} w \delta\right)$.

$S_{1}$ for each fluid pair corresponds to an "effective dissolution rate". Based on the fluid composition, we find $S_{1 \mathrm{~W}}=0.7 \mathrm{kPa}^{-1} \mathrm{~s}^{-1}$ for water, $S_{1 \mathrm{E}}=28.6 \mathrm{kPa}^{-1} \mathrm{~s}^{-1}$ for ethanol, and $S_{1 \mathrm{M}}=103.6 \mathrm{kPa}^{-1} \mathrm{~s}^{-1}$ for methanol [Fig. 5(a)]. Similarly, the coefficient $a_{2}$ is found to linearly depend on $P_{\mathrm{G}}$ with a slope $S_{2 \mathrm{~W}}=0.15 \mathrm{kPa}^{-1} \mathrm{~s}^{-1 / 2}$ for water, $S_{2 \mathrm{E}}=2.70 \mathrm{kPa}^{-1} \mathrm{~s}^{-1 / 2}$ for ethanol, and $S_{2 \mathrm{M}}=6.91 \mathrm{kPa}^{-1} \mathrm{~s}^{-1 / 2}$ for methanol. A linear relationship is found between $S_{1}$ and $S_{2}$ according to $S_{2}=c S_{1}$, with $c=2.5 \times 10^{-2} \mathrm{~s}^{1 / 2}$ [Fig. 5(a), inset]. For practical applications, it would be important to predict the dissolution rate of bubbles directly from the fluid pair composition and pressure $P_{\mathrm{G}}$.

A simple model is developed for estimating $S_{1}$ based on the physicochemical properties of the fluid pair and operating conditions. We assume equilibrium between gas and liquid mass transfer at the interface using Henry's law $P_{\mathrm{G}}=k_{\mathrm{H}} C$, where $k_{\mathrm{H}}$ is the Henry constant between the two fluids, and $C$ is the mass fraction concentration of the gas in the liquid at the interface. The diffusion of $\mathrm{CO}_{2}$ in the liquid also obeys Fick's Law, $J=$ $-\rho D \nabla C$, where $J$ is the mass diffusion flux, $\rho$ is the density, $D$ is the diffusion coefficient, and $\nabla C$ is the mass fraction concentration gradient. ${ }^{30}$ Since the $\mathrm{CO}_{2}$ concentration in the bubble is constant and varies only in the liquid, the concentration gradient for the fast diffusive-mode is assumed to scale as $\nabla C \approx C / \delta$, with $\delta$ being the thickness of the diffusion profile. This argument leads to $J \approx \rho D P_{\mathrm{G}} /\left(k_{\mathrm{H}} \delta\right)$. For elongated bubbles $(d>w)$ in a compact channel, the mass transfer is quasi one-dimensional and occurs primarily across the bubble end-caps so $J \approx-\rho d d l d t=\rho w d f l d t$. Combining the two equations yields $d f l d t \approx D P_{\mathrm{G}} /\left(k_{\mathrm{H}} w \delta\right)$. For fast diffusive modes, $f \approx a_{1} t \approx S_{1} P_{\mathrm{G}} t$, therefore

$$
S_{1} \approx D /\left(k_{\mathrm{H}} w \delta\right)
$$

Assuming the thickness of the diffusion profile $\delta$ is on the order of the interfacial region width $\varepsilon(\delta \approx \varepsilon \approx 1 \mathrm{~nm})$, together with common values of the diffusion coefficient $D$ and Henry's law constant found in the literature (Table 1), we find agreement between our scaling analysis and experimental data with $S_{1}=$ $14 D /\left(k_{\mathrm{G}} w \delta\right)$. This method can be used to estimate the initial behavior of dissolving bubbles in microchannels.

\section{Conclusions}

In this paper, we experimentally study the rate of $\mathrm{CO}_{2}$ mass transfer into water, ethanol, and methanol using microfluidic 
devices. Monodisperse bubbles are generated in a hydrodynamic focusing junction and dissolving bubbles are monitored using high-speed image processing as they travel along a compact microchannel. We demonstrate that the initial bubble size $d_{0}$ is determined by the injection flow rates, $Q_{\mathrm{L}}$ and $Q_{\mathrm{G}}$, and the channel geometry. By contrast, the rate of dissolution of large bubbles $(d>w)$ depends on the fluid pair composition and the gas injection pressure $P_{\mathrm{G}}$. Indeed, experiments carried out at constant pressure $P_{\mathrm{G}}$ for a variety of injection flow rates show little difference in the initial bubble shrinkage rate. For short time-scales $\left(t<10^{-3} \mathrm{~s}\right)$ after fluid initial contact, bubbles display a fast diffusive behavior, which is interpreted as a $\mathrm{CO}_{2}$ concentration gradient localized in the very thin liquid/gas interfacial region. For the three fluid pairs investigated, the initial rate of dissolution is found proportional to the ratio of the diffusion coefficient and Henry's law constant, $D / k_{\mathrm{H}}$. In conclusion, our study shows that gas impregnation and sequestration processes can be rapidly achieved over very short distances using miniature devices.

\section{Acknowledgements}

We have appreciated discussions with Profs. Jon Longtin and John Kincaid.

\section{References}

1 D. D. Meng, T. Cubaud, C.-M. Ho and C.-J. Kim, J. Microelectromech. Syst., 2007, 16, 1403-1410.

2 T. Thorsen, R. W. Roberts, F. H. Arnold and S. R. Quake, Phys. Rev. Lett., 2001, 86, 4163.

3 R. Dreyfus, P. Tabeling and H. Willaime, Phys. Rev. Lett., 2003, 90, 144505 .

4 S. L. Anna, N. Bontoux and H. A. Stone, Appl. Phys. Lett., 2003, 82, 364-366.

5 P. Garstecki, H. A. Stone and G. M. Whitesides, Phys. Rev. Lett., 2005, 94, 164501.

6 M. T. Kreutzer, F. Kapteijn, J. A. Moulijn and J. J. Heiszwolf, Chem. Eng. Sci., 2005, 60, 5895-5916.

7 L. Shui, J. C. T. Eijkel and A. van de Berg, Adv. Colloid Interface Sci., 2007, 133, 2007.

8 S. Y. Teh, R. Lin, L. H. Hung and A. P. Lee, Lab Chip, 2008, 8, $198-$ 220.

9 C. X. Zhao and A. P. J. Middelberg, Chem. Eng. Sci., 2011, 66, 1394 1411.

10 T. Cubaud, U. Ulmanella and C.-M. Ho, Fluid Dyn. Res., 2006, 38, $772-786$.

11 V. S. Ajaev and G. M. Homsy, Annu. Rev. Fluid Mech., 2006, 38, 277307.

12 A. R. Betz and D. Attinger, Int. J. Heat Mass Transfer, 2010, 53, 3683-3691.

13 A. Gunther, M. Jhunjhunwala, M. Thalmann, M. Schmidt and K. Jensen, Langmuir, 2005, 21, 1547-1555.

14 J. Aubin, M. Ferrando and V. Jiricny, Chem. Eng. Sci., 2010, 65, 2065-2093.

15 D. M. Fries and P. R. von Rohr, Chem. Eng. Sci., 2009, 64, 13261335.
16 P. Garstecki, M. J. Fuerstman, H. A. Stone and G. M. Whitesides, Lab Chip, 2006, 6, 437-446.

17 S. van der Graaf, T. Nisisako, C. G. P. H. Schroen, R. G. M. van der Sman and R. M. Boom, Langmuir, 2006, 22, 4144-4152.

18 J. H. Xu, S. W. Li., G. G. Chen and G. S. Luo, AIChE J., 2006, 52, 2254-2259.

19 V. van Steijn, C. R. Kleijn and M. T. Kreutzer, Phys. Rev. Lett., 2009, 103, 214501.

20 M. J. Jensen, H. A. Stone and H. Bruus, Phys. Fluids, 2006, 18, 077103.

21 P. Garstecki, I. Gitlin, W. DiLuzio, G. M. Whitesides, E. Kumacheva and H. A. Stone, Phys. Rev. Lett., 2004, 85, 2649-2651.

22 T. Cubaud and C.-M. Ho, Phys. Fluids, 2004, 16, 4575.

23 T. Cubaud, M. Tatineni, X. Zhong and C.-M. Ho, Phys. Rev. E: Stat., Nonlinear, Soft Matter Phys., 2005, 72, 037302.

24 N. Dietrich, S. Poncin, N. Midoux and H. Z. Li, Langmuir, 2008, 24, 13904-13911.

25 E. Castro-Hernández, W. van Hoeve, D. Lohse and J. M. Gordillo, Lab Chip, 2011, 11, 2023.

26 J. Park, Z. Nie, A. Kumachev and E. Kumacheva, Soft Matter, 2010, 6, 630-634.

27 E. Stride and M. Edirisinghe, Soft Matter, 2008, 4, 2350-2359.

28 F. Takemura and Y. Matsumoto, Chem. Eng. Sci., 2000, 55, 39073917.

29 F. Takemura and A. Yabe, J. Fluid Mech., 1999, 378, 319-334.

30 R. B. Bird, W. E. Stewart, and E. N. Lightfoot, Transport Phenomena, Wiley, New York, 2007.

31 J. J. Heiszwolf, M. T. Kreutzer, M. G. van den Eijnden, F. Kapteijn and J. A. Moulijn, Catal. Today, 2001, 69, 51-55.

32 J. M. van Baten and R. Krishna, Chem. Eng. Sci., 2004, 59, 25352545.

33 N. Shao, A. Garvriilidis and P. Angeli, Chem. Eng. J., 2010, 160, 873881.

34 J. Yue, G. G. Chen, Q. Yuan, L. Luo and Y. Gonthier, Chem. Eng. Sci., 2007, 62, 2096-2108.

35 J. R. Thome, Int. J. Heat Fluid Flow, 2004, 25, 128-139.

36 P. A. Quinto-Su, K. Y. Lim and C.-D. Ohl, Phys. Rev. E: Stat., Nonlinear, Soft Matter Phys., 2009, 80, 047301.

37 C. Sun, E. Can, R. Dijkink, D. Lohse and A. Prosperetti, J. Fluid Mech., 2009, 632, 5-16.

38 S.-L. Chen, C.-T. Lin, C. Pan, C.-C. Chieng and F.-G. Tseng, Microfluid. Nanofluid., 2009, 7, 807-818.

39 A. B. Subramanianm, C. Mejean, M. Abkarian and H. A. Stone, Langmuir, 2006, 22, 5986-5990.

40 P. Poesio, G. P. Beretta and T. Thorsen, Phys. Rev. Lett., 2009, 103, 064501.

41 R. G. Larson, The structure and Rheology of Complex Fluids, Oxford University Press, Inc., New York, 1999.

42 B. Dollet, W. van Hoeve, J. P. Raven, P. Marmottant and M. Versluis, Phys. Rev. Lett., 2008, 100, 034504.

43 T. Cubaud, Phys. Rev. E: Stat., Nonlinear, Soft Matter Phys., 2009, 80, 026307.

44 M. J. W. Frank, J. A. M. Kuipers and W. P. M. van Swaaij, J. Chem. Eng. Data, 1996, 41, 297-302.

45 I. Dalmolin, E. Skovroinski, A. Biasi, M. L. Corazz, C. Dariva and J. Vladimir Oliveira, Fluid Phase Equilib., 2006, 245, 193200.

46 M. Takahashi, Y. Kobayashi and H. Takeuchi, J. Chem. Eng. Data, 1982, 27, 328-331.

47 T. Schnabel, J. Vrabec and H. Hasse, Fluid Phase Equilib., 2005, 233, 134-143.

48 Y. Miyano and I. Fujihara, Fluid Phase Equilib., 2004, 221, 57-62.

49 H. J. Butt, K. Graf, and M. Kapl, Physics and Chemistry of Interfaces, Wiley-VCH Verlag GmbH \& Co., Weinheim, 2006. 